\title{
Obscured Quasars: Finding and Understanding the Most Luminous Active Supermassive Black Holes with SALT
}

\author{
Kevin N. Hainline, Ryan C. Hickox, Christopher Carroll* ${ }^{*}$ \\ Department of Physics and Astronomy, Dartmouth College, Hanover NH 03755 \\ E-mail: kevin.n.hainline@dartmouth.edu, ryan.hickox@gmail.com, \\ Christopher.M.Carroll.GRedartmouth. edu
}

\section{Adam D. Myers, Michael A. DiPompeo}

Department of Physics and Astronomy, University of Wyoming, Laramie WY 82071

E-mail: geordiemyers@gmail.com, mdipompe@uwyo.edu

\section{Laura Trouille}

Center for Interdisciplinary Exploration and Research in Astrophysics (CIERA) and Department of Physics and Astronomy, Northwestern University, Evanston, IL 60208

E-mail: l-trouille@northwestern.edu

\begin{abstract}
The discovery that all massive galaxies contain central supermassive black holes has lead to a great deal of research into how black holes and their host galaxies grow together. As material falls onto a supermassive black hole, an enormous amount of radiation is produced, and it is thought that the radiation from this “active galactic nucleus' (AGN) can significantly impact the evolution of its host galaxy. Using observations from the Southern African Large Telescope (SALT), the Wide-Field Infrared Survey Explorer (WISE), and the Sloan Digital Sky Survey, we have lead a campaign to detect and characterize large samples of the most luminous AGNs, quasars, throughout the universe. Our work focuses on "obscured" quasars, objects where much of the bright UV and optical light produced near the black hole is blocked by dust along our line of sight to the center of these galaxies, allowing us to observe their host galaxies in detail. By finding and exploring these objects, we can understand the delicate relationship between black hole and stellar growth in galaxies both nearby and at large cosmic distances.
\end{abstract}

SALT Science Conference 2015

1-5 June 2015

Stellenbosch Institute of Advanced Study, South Africa

\footnotetext{
${ }^{*}$ Speaker.

${ }^{\dagger}$ These observations were partially supported by NASA through ADAP award NNX12AE38G and by the National Science Foundation through grand numbers 1211096 and 1211112.
} 


\section{Introduction}

The role of quasars in the context of galaxy evolution is not well understood. Quasars have been theorized to influence the interstellar gas content and gas temperature throughout their host galaxies, which may lead to reduced star formation [1,2]. Unobscured quasars, where powerful emission from near the accreting supermassive black hole is viewed directly, are relatively straightforward to detect in optical surveys, but a complete census of quasars requires observations of the obscured population, where nuclear and galaxy-scale dust occults sightlines to the central black hole. Obscured quasars have been selected from their narrow optical emission lines [3,4], but with the launch of the Spitzer and Wide Field Infrared Survey Explorer (WISE) space telescopes, we can use observations made in the infrared where dust near the central black hole reprocesses quasar emission, producing a characteristic red IR power-law spectrum. Infrared color criteria, then, have been proposed to efficiently select obscured quasars [5,6,7,8,9,10,11], and we performed a blind survey to select these objects from WISE photometry using observations with the Southern African Large Telescope (SALT).

\section{Assembling a sample of obscured quasar candidates}

We selected our candidate obscured quasars using Sloan Digital Sky Survey (SDSS) and WISE data, targeting objects in Stripe $82\left(-2^{\circ}<\mathrm{DEC}<2^{\circ}\right)$. To obtain the signal-to-noise in the spectra required for observing the strong optical emission lines characteristic of quasar activity, we limited our objects to $20<g<22$ (in $\mathrm{AB}$ magnitudes), and objects were chosen without existing SDSS spectra. We furthermore separated our targets into two groups based on optical-to-IR color in order to test an obscured quasar criteria used in Hickox et al. [12]. Our WISE color-color plot used for selecting our targets is shown in Figure 1. Spectral energy distribution (SED) modeling of our sources revealed a variety of host galaxy types and underlying quasar luminosities. We selected some objects to have best-fit SEDs indicative of unobscured quasar activity to further refine the color selection criteria. Our sample, which consisted of forty targets, was observed with the Robert Stobie Spectrograph (RSS) on SALT in 2012-2013 under Proposals 2012-2-DC-002, 2013-1-DC003, 2013-2-DC-003.

The spectra show a range of optical emission lines, and due to the wavelength coverage of SALT RSS, we primarily observed Mg II $\lambda$ 2798, [NeV] $\lambda 3427$, [OII] $\lambda \lambda 3726,3729$, [NeIII] $\lambda 3869, \mathrm{H} \beta$, and [OIII] $\lambda \lambda 4959,5007$ in our objects. We were able to identify emission features, and therefore estimate a redshift, for 35 out of the 40 objects in our sample. For the five remaining objects, there were no features in the spectra allowing for reliable redshift indicators. The average redshift of the objects in our sample is $\langle z\rangle=0.52$, which agrees with what would be expected both from the sensitivity of WISE [14] as well as the optical photometry requirements for observations with SALT. Two of our objects have redshifts at $z>2$. One of these objects is a broad absorption line quasar with strong $\mathrm{Ly} \alpha, \mathrm{NV}$, and CIV in emission with blueshifted absorption, and the other is a quasar with $\operatorname{Ly} \alpha$ (with central self-absorption) and CIV in emission.

\section{Tracers of obscured activity}

For the majority of the sample, due to the redshifts of the objects, we could not use the most 


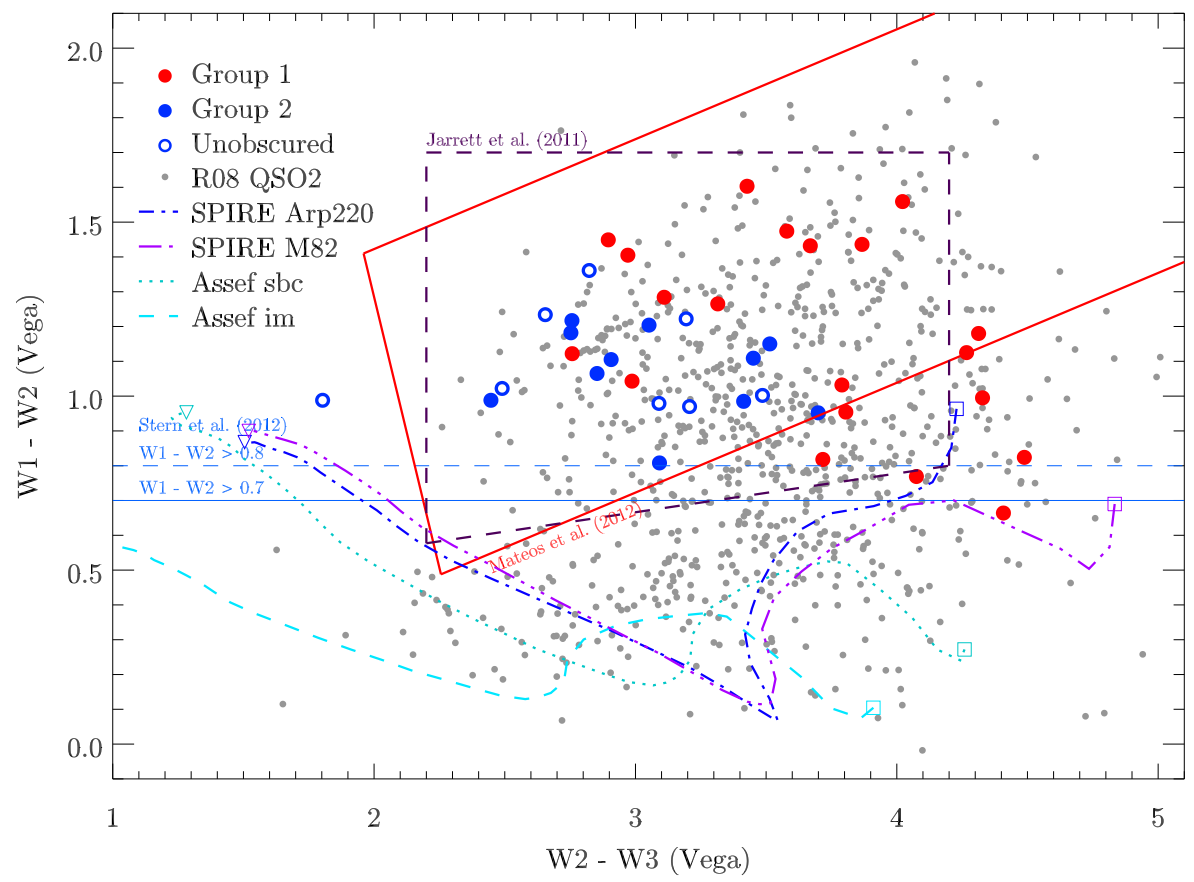

Figure 1: WISE color-color plot showing the selection of our quasar candidates. Our targets, split into two groups based on optical-IR color, are plotted with red and blue circles. We show the common WISE quasar selection criteria from Stern et al. [7] with blue horizontal dashed and solid lines. We plot the confirmed obscured quasars from Reyes et al. [4] with grey points. Finally, we plot tracks showing how star-forming galaxy templates move on this diagram as a function of redshift, from $z=0$ (square) to $z=2$ (downward-facing triangle). This figure is reproduced from Hainline et al. [13].

common optical emission lines that indicate quasar activity, as they are redshifted off of the RSS detector. Instead, we used the emission line ratio of [NeIII] $\lambda 3869$ to [OII] $\lambda \lambda 3726$, which has been shown, along with rest-frame $g-z$ color, to separate luminous quasars from star-forming galaxies (the "TBT diagram"; [15]). We show our candidates where we detected both [NeIII] $\lambda 3869$ to [O II] $\lambda \lambda 3726$ on the TBT diagram in Figure 2. As can be seen from the position of the objects on the figure, the majority of our objects have line ratios and colors indicating strong quasar activity. In addition, our extensive SED modeling for the sample required quasar templates to accurately reproduce the infrared emission from these sources. Galaxy templates, even those with extreme star-forming properties such as Arp 220 and M82, could not replicate the steep infrared power-law emission observed in our sources. In addition, the SED modeling demonstrates the wide range in quasar obscuration probed by our color selection criteria. Together, these results indicate the efficacy in using infrared colors to select powerful obscured quasars.

\section{Conclusions}

These observations have served to focus future observations of obscured populations. As seen in Figure 1, the infrared selection criteria of Mateos et al. [16] omits objects with very red WISE 


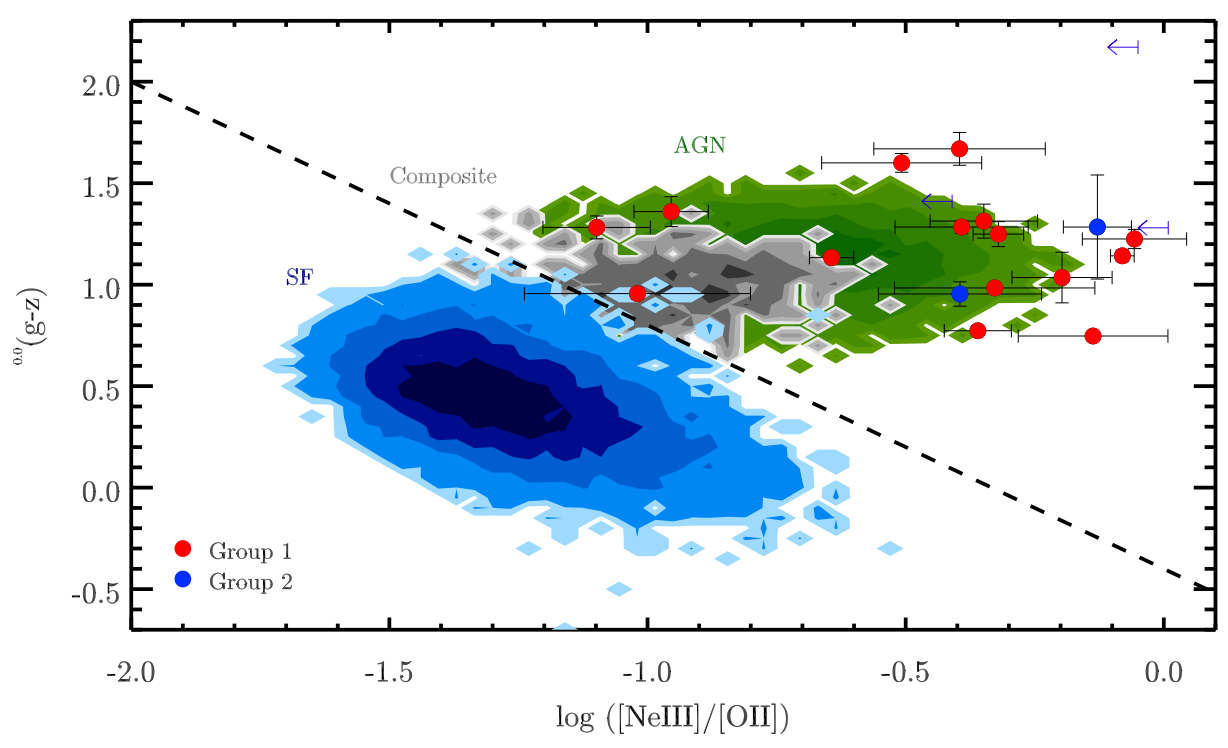

Figure 2: Emission-line diagnostic diagram from Trouille et al. [15]. The objects in our sample with significant detections of [NeIII] $\lambda 3869$ to [OII] $\lambda \lambda 3726$ are plotted with red and blue points, depending on their selection group, as in Figure 1. We plot SDSS-selected galaxies from Trouille et al. [15] with colored contours, in blue we plot emission-line selected star forming galaxies, in green we plot emission-line selected active galactic nuclei, and composite objects are plotted in grey. The objects that comprise our sample have emission lines strongly indicating quasar activity. This figure is reproduced from Hainline et al. [13].

W2-W3 colors, as this region is contaminated by extreme local star star-forming galaxies. Five out of six of of the galaxies that fell outside of the Mateos et al. selection wedge in our sample were confirmed, from their spectroscopy, to host quasars which were among the most obscured in our sample, with high optical extinction as estimated from SED fitting. These objects are excellent targets for further follow-up observations with hard X-ray telescopes, such as the Nuclear Spectroscopic Telescope Array (NuSTAR). These highly obscured quasars may represent an important stage in the lifetime of a galaxy, where we are seeing a quasar in an early, highly obscured phase following a major merger. As such, SALT observations to explore the optical spectroscopic properties of these obscured quasars are being obtained to help shed light on this phase in galaxy evolution.

Our results demonstrate the power in using optical+IR photometry to uncover obscured quasar activity. We selected quasar candidates only through their photometry, and then used SALT RSS to determine whether or not they hosted a quasar. The vast majority of the objects we targeted had strong evidence from both their observed emission lines and their SEDs for obscured quasar activity, with redshifts of $z<1$ on average. Future large optical surveys, such as what will be undertaken by the forthcoming Large Synoptic Survey Telescope, will, along with WISE photometry and SALT spectroscopy, uncover a large population of obscured quasars even out to larger redshifts. 


\section{References}

[1] Fabian, A. C. et al. 2012, ARA\&A, 50, 455

[2] Alexander, D. M. et al. 2012, NewAR, 56, 93

[3] Zakamska, N. L. et al. 2003, AJ, 126, 2125

[4] Reyes, R. et al. 2008, AJ, 136, 2373

[5] Lacy, M. et al. 2004, ApJS, 154, 166

[6] Stern, D. et al. 2005, ApJ, 631, 163

[7] Stern, D. et al. 2012, ApJ, 753, 30

[8] Donley, J. L. et al. 2008, ApJ, 687, 111

[9] Donley, J. L. et al. 2012, ApJ, 748, 142

[10] Ashby, M. L. N. et al. 2009, ApJ, 701, 428

[11] Assef, R. J. et al. 2010, ApJ, 713, 970

[12] Hickox, R. C. et al. 2007, ApJ, 671, 1365

[13] Hainline, K. N. et al. 2014, ApJ, 795, 124

[14] Assef, R. J. et al. 2013, ApJ, 772, 26

[15] Trouille, L. et al. 2011, ApJ, 742, 46

[16] Mateos, S. et al. 2012, MNRAS, 426, 3271 